Synthesis Alerts is a monthly feature to help readers of Synthesis keep abreast of new reagents, catalysts, ligands, chiral auxiliaries, and protecting groups which have appeared in the recent literature. Emphasis is placed on new developments but established reagents, catalysts etc are also covered if they are used in novel and useful reactions. In each abstract, a specific example of a transformation is given in a concise format designed to aid visual retrieval of information.

Synthesis Alerts is a personal selection by:

Elyse Bourque, Jennifer Delaney, Andrew Gunn, Stephen McAteer, Stefan Schunk and Josephine Yuen, Department of Chemistry, Leeds University, Leeds, LS2 9JT, UK.

Georg Thieme Verlag does not accept responsibility for the accuracy, content, or selection of the data.

Synthesis 2002, No. 13, 2009 2002. Article Identifier:

1437-210X,E;2002,0,13,1937,1944,ftx,en;X01302SS.pdf.

(C) Georg Thieme Verlag Stuttgart · New York

ISSN 0039-7881
The journals regularly covered by the abstractors are:

Angewandte Chemie International Edition

Bulletin of the Chemical Society of Japan

Chemical Communications

Chemistry A European Journal

Chemistry Letters

Collection Czechoslovak Chemical Communications

European Journal of Organic Chemistry

Helvetica Chimica Acta

Heterocycles

Journal of the American Chemical Society

Journal of Organic Chemistry

Organic Letters

Organometallics

Perkin Transactions 1

Synlett

Synthesis

Tetrahedron

Tetrahedron Asymmetry and Tetrahedron Letters
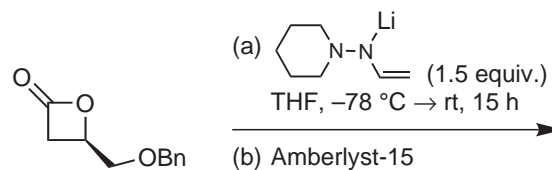

(b) Amberlyst-15 THF, $\Delta, 3 \mathrm{~h}$

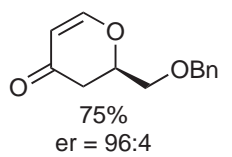

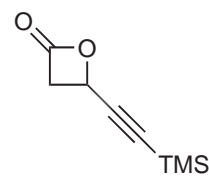

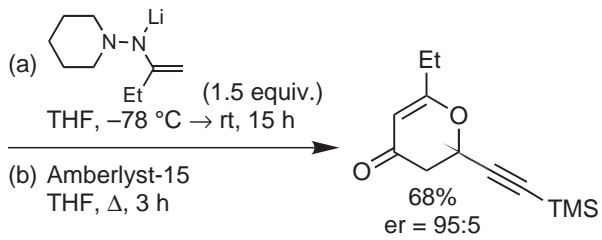

9 Examples (yields $68-81 \%$, \%ee $90-91 \%$ ).

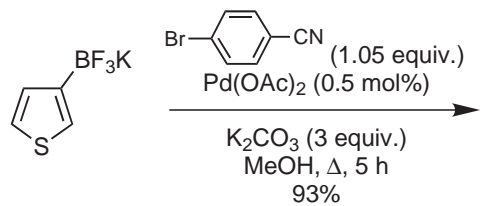

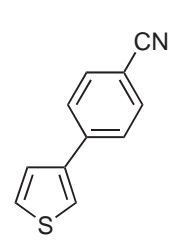

23 Examples (yields 52-99\%).

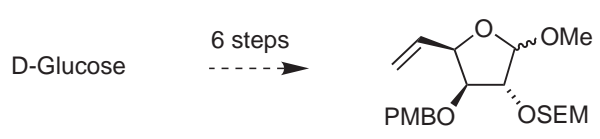

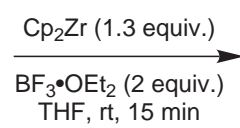

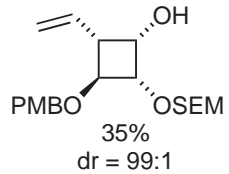




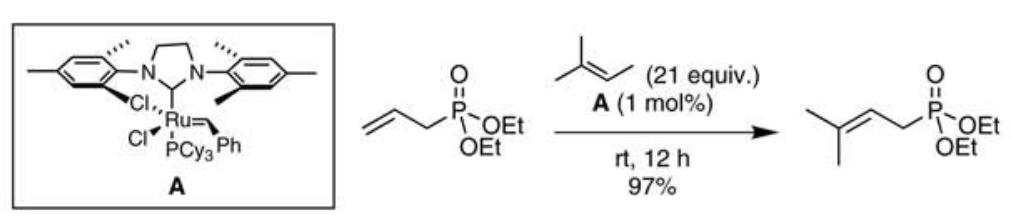

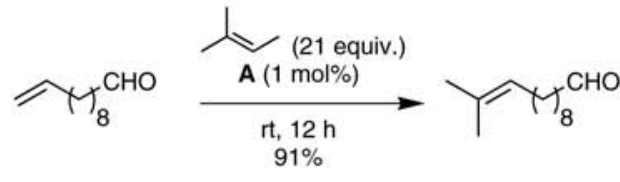

13 Examples (yields 48-99\%)<smiles>CC(C)=CCOC(=O)C=Cc1ccccc1</smiles>

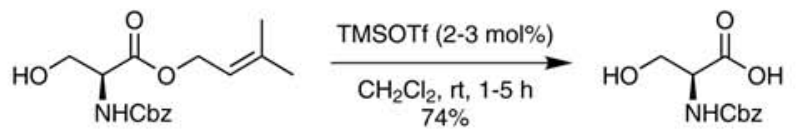

Synthesis of 3-aryl- $\delta$-lactones via asymmetric Michael reactions.

Smitrovich, J. H.; Boice, G. N.; Qu, C.; DiMichele, L.; Nelson, T. D.; Huffman,

Asymmetric 1,4-Addition/Lactonization

M. A.; Murry, J.; McNamara, J.; Reider, P. J. Org. Lett. 2002, 4, 1963.

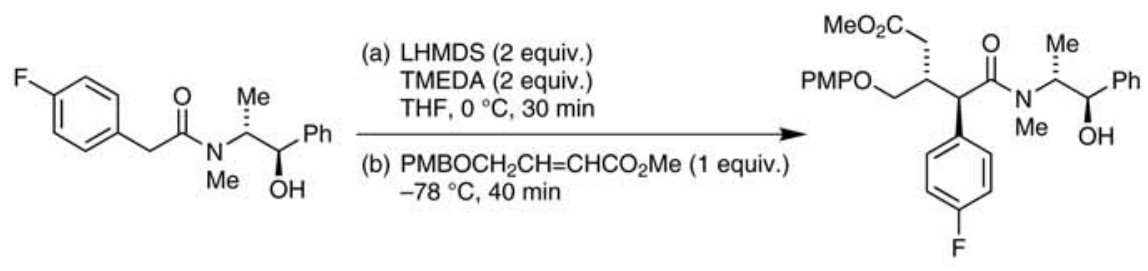

(a) $\mathrm{LiBH}_{4}$ (1.3 equiv.) $\mathrm{PhMe}, \mathrm{rt}$, overnight

(b) $\mathrm{HCl}$ ( 1.5 equiv.)

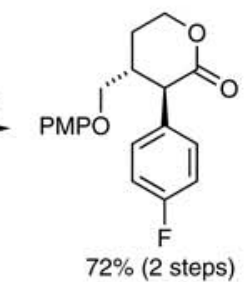

9 Examples (yields $46-89 \%$, \%ee $76-93 \%$ ).

The $[2+1]$ and $[4+3]$ cyclization reactions of fulvenes with Fisher carbene complexes.

Barluenga, J.; Martinez, S.; Suarez-Sobrino, A. L.; Tomas, M. J. Am. Chem. Soc. 2002, 124, 5948.<smiles>CC1=CCC2CCCC1C(=O)C2</smiles>

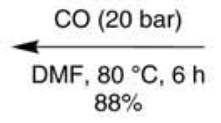<smiles>COC(C#CPc1ccccc1)=[N+]([O-])OC</smiles><smiles>CC(C)=C1C=CC=C1</smiles>

1.2 equiv.

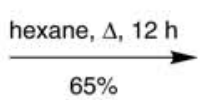

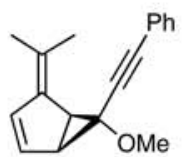

$3: 1$

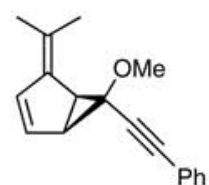

5 Examples of [4+3] cyclization (yields 50-88\%). 4 Examples of [2+1] cyclization (yields 65-73\%).

Three-component cycloadditions: the first transition metal-catalyzed $[5+2+1]$ cycloaddition reactions.
Wender, P. A.; Gamber, G. G.; Hubbard, R. D.; Zhang, L. J. Am. Chem. Soc. 2002, 124, 2876.

Three-component cycloadditions: the first transition metal-catalyzed [5+2+1] cycloaddition reaction
Wender, P. A.; Gamber, G. G.; Hubbard, R. D.; Zhang, L. J. Am. Chem. Soc. 2002, 124, 2876.

$[5+2+1]$ Cycloaddition
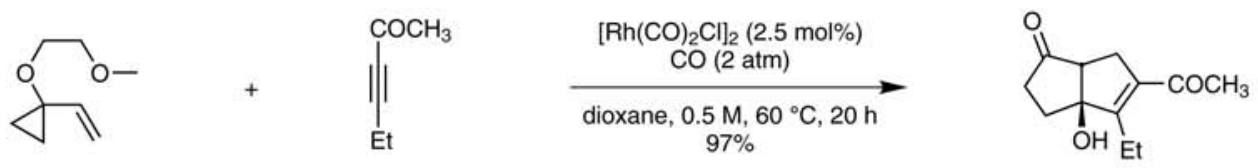

1.2 equiv.

9 Examples (yield 48-97\%). 


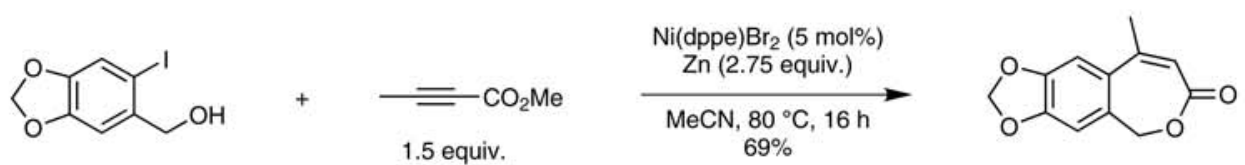

13 Examples (yield 56-81\%).
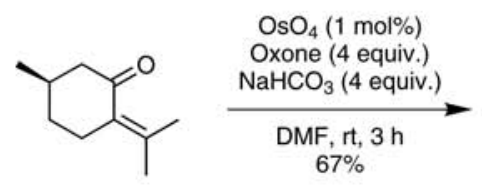<smiles>C[C@H](CCC(=O)O)CC(=O)O</smiles>
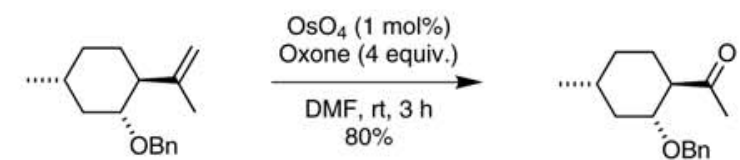

24 Examples (yield 50-97\%).

A recyclable Ru catalyst for enantioselective olefin metathesis in air.

Van Veldhuizen, J. J.; Garber, S. B.; Kingsbury, J. S.; Hoveyda, A. H. J. Am. Chem. Soc. 2002, 124,

Metathesis

4954.
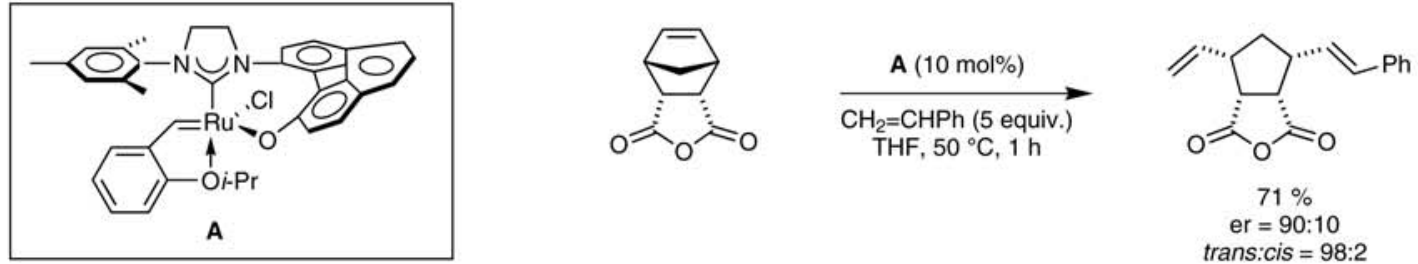

4 Examples (yield $57-76 \%$, \%ee $80-98 \%$ ).

Enantioselective addition of terminal alkynes to imines catalyzed by copper(I)pybox complex.

Wei, C.; Li, C.-J. J. Am. Chem. Soc. 2002, 124, 5638.

Asymmetric 1,2-Addition
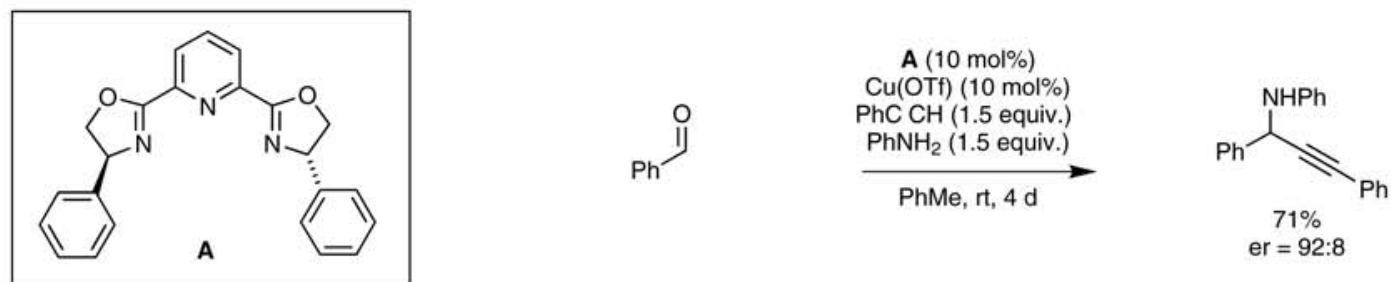

11 Examples (yield 63-93\%, \%ee 82-96\%). Water can also be used as solvent.

Catalytic asymmetric Mannich-type reactions in aqueous media.

Kobayashi, S.; Hamada, T.; Manabe, K. J. Am. Chem. Soc. 2002, 124, 5640.

Asymmetric 1,2-Addition

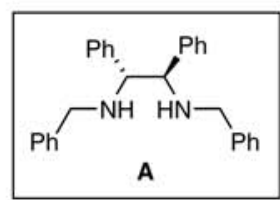<smiles>CCOC(=O)/C=N/NC(=O)c1ccccc1</smiles><smiles>C=C(OC)c1ccc(C)cc1</smiles>

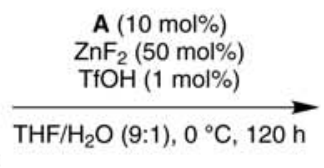

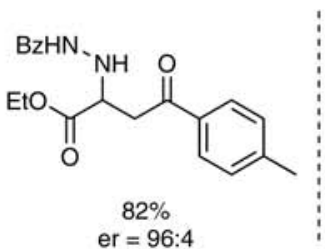<smiles>CCC(C)NC(=O)NC(CO)CC(O)c1ccccc1</smiles> 
Solution- and solid-phase synthesis of heterocycle and enediyne libraries.

Nicolaou, K. C.; Montagnon, T.; Ulven, T.; Baran, P. S.; Zhong, Y.-L.; Sarabia, F. J. Am. Chem. Soc. $2002,124,5718$.<smiles>OC1(Cc2ccccc2)CCCCCCCCCC1Cc1ccccc1</smiles>

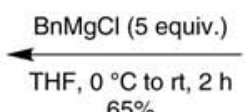
$65 \%$

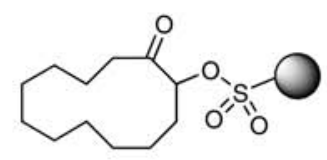

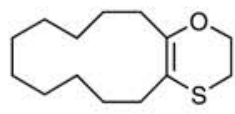

20 Examples of functionalizing-cleavage options (yields 38-95\%). 4 Examples of functionalizing-cleavage options involving carbon-carbon bond-forming (yields $65-85 \%$ ).

Catalytic asymmetric $\alpha$-amination of aldehydes.

List, B. J. Am. Chem. Soc. 2002, 124, 5656.

Asymmetric 1,2-Addition $\alpha$-Amination

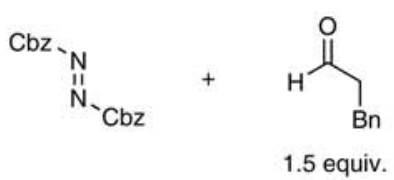

(a) (S)-proline (10 mol\%) $\mathrm{MeCN}, 0^{\circ} \mathrm{C} \rightarrow \mathrm{rt}, 3 \mathrm{~h}$

(b) $\mathrm{NaBH}_{4}$ (1 equiv.) $\mathrm{EtOH}, 0^{\circ} \mathrm{C}, 5 \mathrm{~min}$

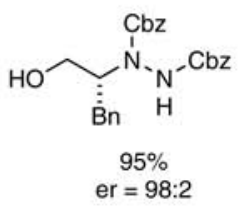<smiles>O=C1NC(Br)CO1</smiles>

$(R)$-Evans auxiliary

5 Examples (yield 93-99\%, \%ee 95-97\%). The synthesis of Evans auxiliary is presented.

Catalytic hetero-Heck type reaction.

Helaja, J.; Göttlich, R. Chem. Commun. 2002, 720.

Cyclization

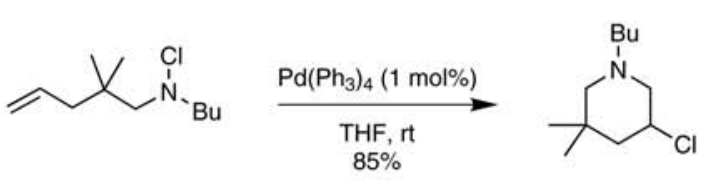<smiles>C=CCN(Cl)CC(C)(C)CC=C</smiles>

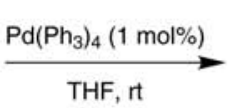<smiles>C=CCN1CC(Cl)CC(C)(C)C1</smiles>

8 Examples (yields $43-86 \%$ ).

Tandem allylic acetate isomerisation-ring closing metathesis.

Braddock, D. C.; Matsuno, A. Tetrahedron Lett. 2002, 43, 3305.

Isomerisation / Metathesis
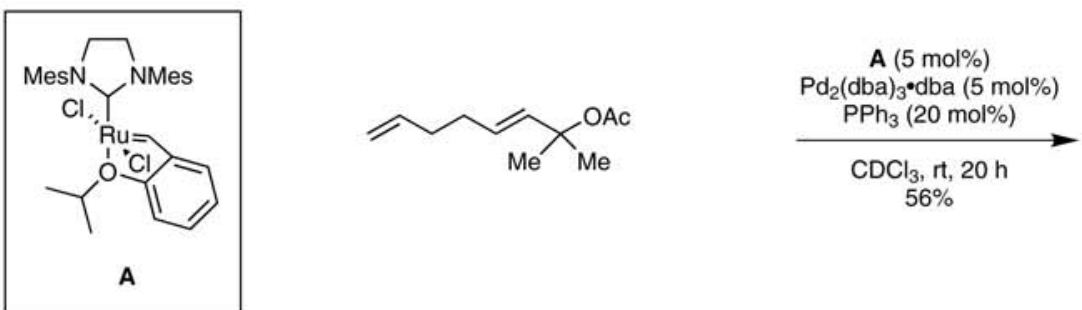

$\underset{56 \%}{\underset{\mathrm{CDCl}_{3}, \mathrm{rt}, 20 \mathrm{~h}}{\mathrm{PPh}_{3}(20 \mathrm{~mol} \%)}}$<smiles>COC1CCCCC1</smiles>

3 Examples (yields trace- $56 \%$ ). Development of the catalyst and other metathesis reactions are also reported.

Dynamic kinetic asymmetric transformation / kinetic resolution.

Hughes, D. L.; Palucki, M.; Yasuda, N.; Reamer, R. A.; Reider, P. J. J. Org. Chem. 2002, 67, 2762.

Asymmetric Allylic Alkylation

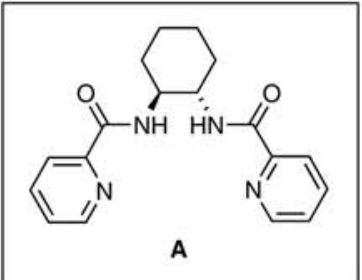<smiles>C=C[C@H](OC(C)=O)c1ccccc1</smiles>

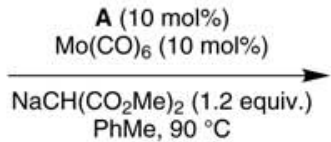<smiles>C=CC(C(=O)OC)C(C(=O)OC)c1ccccc1</smiles>

er $=99: 1$

3 Examples. Effect of solvent and temperature upon the reaction kinetics was also investigated. 
Iodolactonization and cyclopropanation applied to the synthesis of Constanolactones $\mathrm{A}$ and $\mathrm{B}$

Yu, J.; Lai, J.-Y.; Ye, J.; Balu, N.; Reddy, L. M.; Duan, W.; Fogel, E. R.; Capdevila, J. H.; Falck, J. R.

lodolactonization/Alkylation Tetrahedron Lett. 2002, 43, 3939 .<smiles>CC1(C)OCC(C(/C=C/CCCC(=O)O)[C@H](Oc2ccccc2)c2ccccc2)O1</smiles>

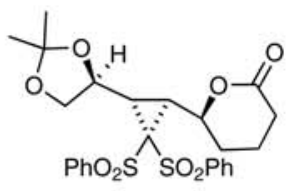

THF, $68^{\circ} \mathrm{C}, 44 \mathrm{~h}$

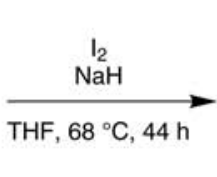

\section{9 steps}

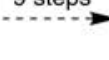<smiles>O=C1CCC[C@H](/C=C/[C@H](O)/C=C/[C@H](O)C/C=C/O)O1</smiles>

Constanolactone A

Constanolactone B was also prepared using the same method.

Stereoselective synthesis of syn- and anti-1,3- and 1,2-dimethyl arrays.

Williams, D. R.; Kissel, W. S.; Li, J. J.; Mullins, R. J. Tetrahedron Lett. 2002, 43, 3723.

Asymmetric 1,4-Addition

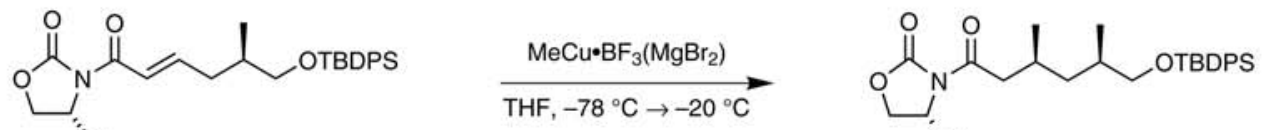

" $\mathrm{Ph}$ $\mathrm{Ph}$<smiles>CC(/C=C/C(=O)N1CCOC1=O)CO[R5]#[SH]</smiles>

$\mathrm{Ph}$<smiles>CC(CO[13CH3])C[C@H](C)CC(=O)N1CCOC1=O</smiles>

$\mathrm{Ph}$

13 Examples (yields $75-99 \%$, \%de 0-99\%).

Rh-catalyzed isomerization of 4-alkynals to dienals.

Tanaka, K.; Fu, G. C. Chem. Commun. 2002, 684.

Isomerization$$
\text { Tanaka, K.; Fi, G. C. Chem. Commun. } 2002,684 .
$$

$\overbrace{\mathrm{Ph}}^{\mathrm{TMS}} \mathrm{CHO}$

[Rh(BINAP) $] \mathrm{BF}_{4}(5-10 \mathrm{~mol} \%)$

$\mathrm{CH}_{2} \mathrm{Cl}_{2}, \mathrm{rt}, 1 \mathrm{~d}$
$87 \%$
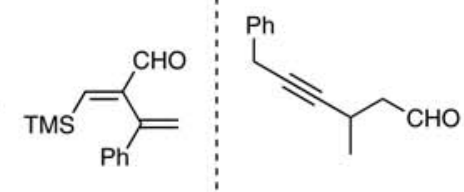

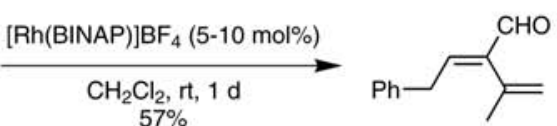

5 Examples (yields 50-87\%).

Alkylidene carbene $1,5-\mathrm{CH}$ insertion reactions of $\alpha$-amino acid derived substrates.

Mapitse, R.; Hayes, C. J., Tetrahedron Lett. 2002, 43, 3541.

CH Insertion
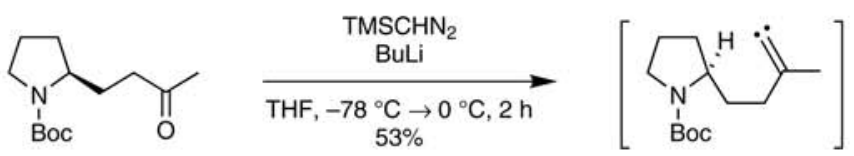

$1,5-\mathrm{CH}$ insertion

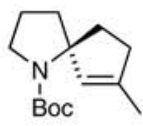

5 Examples (yields 10-53\%).

Asymmetric conjugate addition of Li[RCul]/TMSI to $\mathrm{N}$-enoyl oxazolidinones.

Pollock, P.; Dambacher, J.; Anness, R.; Bergdahl, M. Tetrahedron Lett. 2002, 43, 3693.

Asymmetric 1,4-Addition

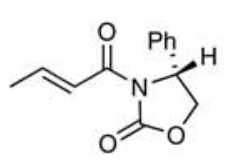
(a) $\mathrm{PhLi}$ ( 1.6 equiv.)
Cul (1.9 equiv.)
TMSI (1.6 equiv.)
THF, $-78^{\circ} \mathrm{C}, 4 \mathrm{~h}$
(b) $\mathrm{Et}_{3} \mathrm{~N}, \mathrm{NH}_{4} \mathrm{Cl},(\mathrm{aq}),-78^{\circ} \mathrm{C}, 1 \mathrm{~h}$

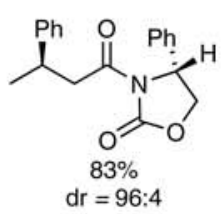

24 Examples (yields 0-96\%, \%de 0-96\%). The use of valine and phenylala nine-derived oxazolidinones is also reported. 

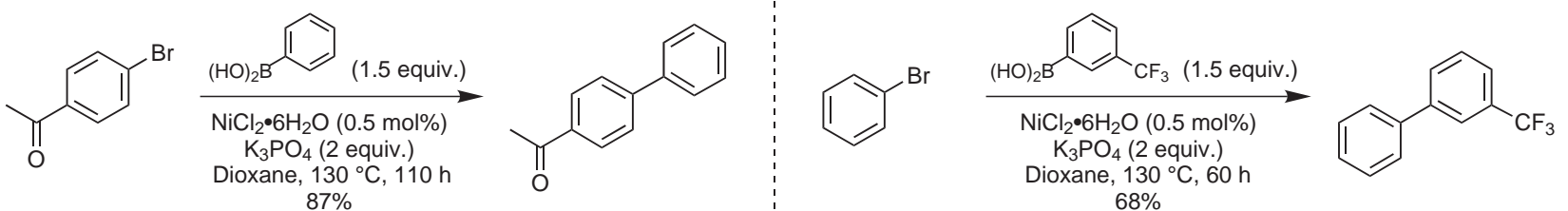

11 Examples (yields 6-87\%).

Cleavage of PMP, THP and 1,3-dithiane protecting groups by Selectfluor ${ }^{\mathrm{TM}}$.

Liu, J.; Wong, C.-H. Tetrahedron Lett. 2002, 43, 4037.

Deprotection
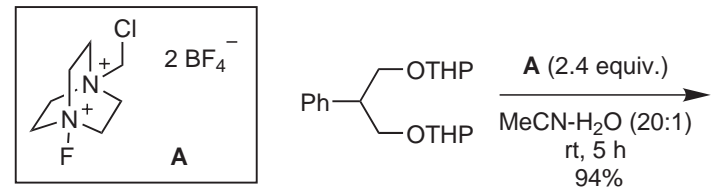<smiles>OCC(CO)c1ccccc1</smiles><smiles>CC(=O)C1CC2SCCS21[O-]</smiles>
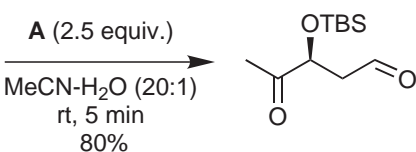

$80 \%$

4 Examples of PMP removal (yields 87-92\%), 5 examples of THP removal (yields 89-95\%) and 5 examples of 1,3-dithiane (yields 80-95\%).

Tandem ene-reaction/intramolecular Sakurai cyclization.

Marko, I. E.; Dumeunier, R.; Leclercq, C.; Leroy, B.; Plancher, J.-M.; M ekhalfia, A.; Bayston, D. J. Synthesis,

Heteroannulation 2002, 958.<smiles>C=C1CC(C(C)C)OC1CC(C)O</smiles><smiles>CCC(C)OC(C)CC(=O)CC(C)C</smiles><smiles></smiles>

(a) NBS (1 equiv.)

(b) TBAF (2 equiv.) $\mathrm{CH}_{2} \mathrm{Cl}_{2}$, rt, 5 min

(c) $\mathrm{MnO}_{2}$ (20 equiv.) $\mathrm{KCN}$ (1 equiv.)

$\mathrm{MeCN}, 0^{\circ} \mathrm{C}, 25 \mathrm{~min}$

$61 \%$

Ene adducts (8 examples, yields 51-79\%), tetrahydropyrans (5 examples, yields 57-85\%), $\gamma$-butyrolactones (9 examples, yields 57-99\%).

Regiospecific Pd-catalyzed hydrocarbonation at $\alpha$-position of a Michael acceptor.

Shim, J.-G.; Park, J. C.; Cho, C. S.; Shim, S. C.; Yamamoto, Y. Chem. Commun. 2002, 852.

Hydrocarbonation<smiles>Cc1ccc(C=O)cc1</smiles>
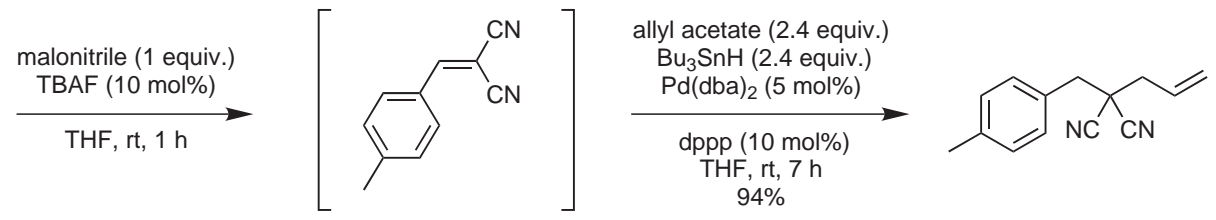

11 Examples (yields 72-97\%). A one-pot variant via in situ generation of the Michael acceptor (4 examples, yields 70-94\%) is als o reported.

$\mathrm{Cs}_{2} \mathrm{CO}_{3}$-Promoted synthesis of carbonates and carbamates via a 3 component coupling.

Salvatore, R. N.; Chu, F.; Nagle, A. S.; Kapxhiu, E. A.; Cross, R. M.; J ung, K. W. Tetrahedron, $2002,58,3329$.

3 Component Coupling
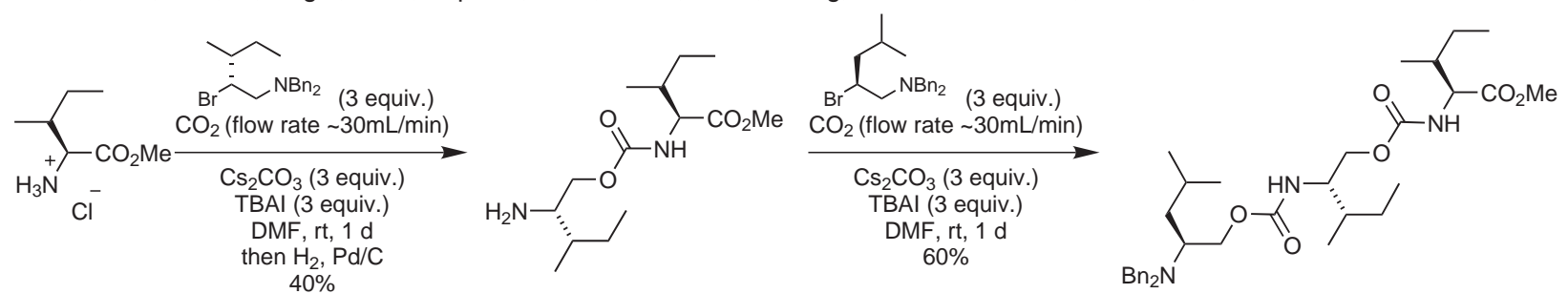

Solution phase: carbonates (16 examples, yields 65-98\%), carbamates ( 44 examples, yields 47-98\%).

Solid phase: carbonates (14 examples, yields 63-99\%), carbamates (30 examples, yields 21-97\%) 


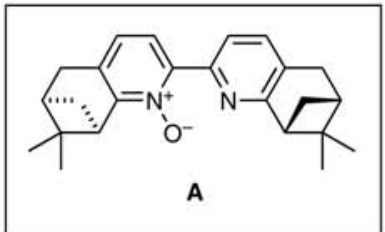

1.1 equiv.
$\mathrm{R}$

11 Examples (yields 10-85\%, \%ee 4-92\%).

Asymmetric addition of diethylzinc to aromatic aldehydes catalyzed by fluorous BINOL-Ti complexes.

Nakamura, Y.; Takeuchi, S.; Okumura, K.; Ohgo, Y.; Curran, D. P. Tetrahedron 2002, $58,3963$.

Asymmetric 1,2-Addition<smiles>[R5][SiH2]c1ccc2c(-c3c(O)ccc4cc([Si])ccc34)c(O)ccc2c1</smiles><smiles>O=CBr</smiles>

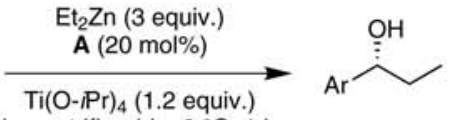

\begin{tabular}{lrr} 
Ar & Yield & $\%$ ee \\
\hline $\mathrm{Ph}$ & $92 \%$ & $84 \%$ \\
$3-\mathrm{MeO}-\mathrm{C}_{6} \mathrm{H}_{4}$ & $95 \%$ & $85 \%$ \\
$4-\mathrm{Me}-\mathrm{C}_{6} \mathrm{H}_{4}$ & $91 \%$ & $81 \%$ \\
1-Naphthyl & $98 \%$ & $91 \%$ \\
4-Cl- $\mathrm{C}_{6} \mathrm{H}_{4}$ & $93 \%$ & $82 \%$
\end{tabular}

9 Examples (yields $91-98 \%$, \%ee $78-91 \%$ ). Almost complete recovery of catalyst after reaction.

Cu-catalyzed aerobic oxidation of alcohols using fluorous biphasic catalysis.

Ragagnin, G.; Betzmeier, B.; Quici, S.; Knochel, P. Tetrahedron 2002, 58, 3985.

Oxidation
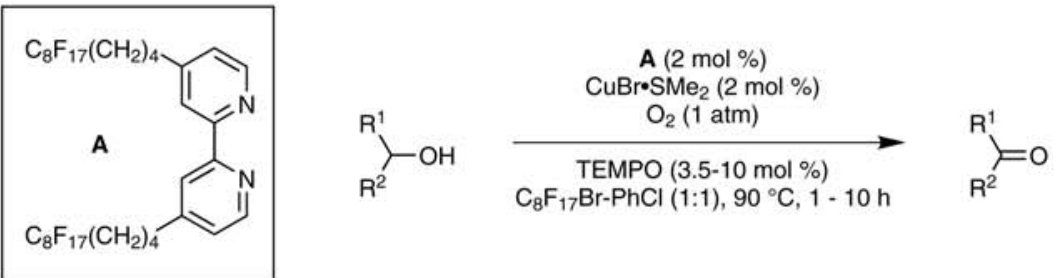

\begin{tabular}{lll}
$\mathrm{R}^{1}$ & $\mathrm{R}^{2}$ & Yield \\
\hline$p-\mathrm{NO}_{2} \mathrm{Ph}$ & $\mathrm{H}$ & $93 \%$ \\
$\mathrm{CH}_{3}\left(\mathrm{CH}_{2}\right)_{8}$ & $\mathrm{H}$ & $73 \%$ \\
$p-\mathrm{NCPh}_{3}$ & $\mathrm{CH}_{3}\left(\mathrm{CH}_{2}\right)_{3}$ & $95 \%$ \\
$\mathrm{CH}_{3}\left(\mathrm{CH}_{2}\right)_{7}$ & $\mathrm{CH}_{3}$ & $88 \%$
\end{tabular}

21 Examples (yields 31-97\%). Selective oxidation of cis:trans mixtures of substituted cyclohexanols (6 examples) is also reported.

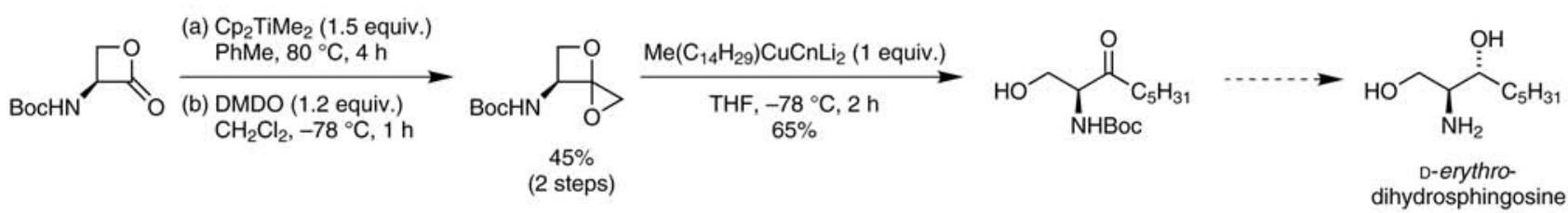

The total syntheses of D-erythro-dihydrosphingosine and D-xylo-phytosphingosine are reported.

Conjugate addition and intramolecular acylation of an amino ester with an acetylinic sulfone.

Back, T. G.; Hamilton, M. D. Org. Lett. 2002, 4, 1779.

Conjugate Addition/

Intramolecular Acylation<smiles>COc1ccc(C#C[As])cc1OC</smiles><smiles>COc1ccc(C(=C[As])N2CCCC[C@H]2CC(C)=O)cc1OC</smiles>
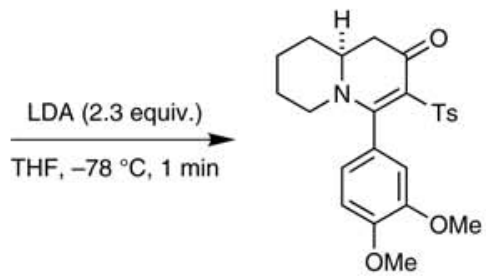

The remaining steps towards the synthesis of lasubine II are reported. 
A one-pot access to cyclopropanes from allylic ethers via hydrozirconation-deoxygenative ring formation. Gandon, V.; Szymoniak, J. Chem. Commun. 2002, 1308.

Cyclopropanation<smiles>C=C(c1ccccc1)C(OC)/C(C)=C/c1ccccc1</smiles>
(a) $\mathrm{Cp}_{2} \mathrm{Zr}(\mathrm{H}) \mathrm{Cl}(1.5$ equiv.) $\mathrm{PhH}, 60^{\circ} \mathrm{C}, 2 \mathrm{~h}$
(b) $\mathrm{BF}_{3} \cdot \mathrm{OEt}_{2}(1.6$ equiv.) $\mathrm{PhH}, 0^{\circ} \mathrm{C} \rightarrow \mathrm{rt}, 1 \mathrm{~h}$

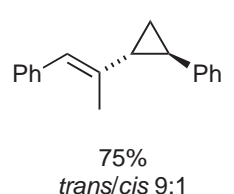

$\overbrace{P h}^{O B n}$
(a) $\mathrm{Cp}_{2} \mathrm{Zr}(\mathrm{H}) \mathrm{Cl}$ ( 1.5 equiv.) $\mathrm{PhH}, 60^{\circ} \mathrm{C}, 1.5 \mathrm{~h}$
(b) $\mathrm{BF}_{3} \cdot \mathrm{OEt}_{2}(1.6$ equiv.) $\mathrm{PhH}, 0^{\circ} \mathrm{C} \rightarrow \mathrm{rt}, 1 \mathrm{~h}$

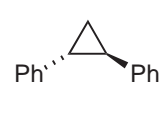

$60 \%$ trans/cis $=100: 1$

11 Examples (yields 42-92\%, 71:29 trans/cis 100:1).

Diastereoselective hetero-Diels-Alder reactions.

Joly, G. D.; Jacobsen, E. N. Org. Lett. 2002, 4, 1795.

[4+2] - Cycloaddition

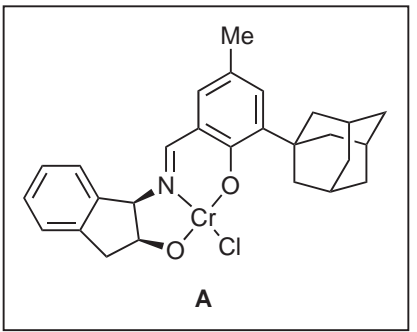<smiles>C=C(C)C=COC</smiles><smiles>CC(O[Sb])C(=O)c1ccccc1</smiles>

(a) A (5 mol\%)

$\mathrm{BaO}$ (1.3 equiv.) EtOAc, $4^{\circ} \mathrm{C}, 1 \mathrm{~d}$

(b) TFA

1.2 equiv.

$\mathrm{CH}_{2} \mathrm{Cl}_{2}, 4^{\circ} \mathrm{C} \rightarrow \mathrm{rt}, 10 \mathrm{~min}$<smiles>C[C@H]([OH2+])[C@H]1CC(=O)C=CO1</smiles>

5 Examples (yields 58-97\%, \%ee 99\%, \%de 56-94\%).

Control of 2-alkenylfuran (E/Z)-configuration.

Wipf, P.; Soth, M. J. Org. Lett. 2002, 4, 1787.

Heteroannulation

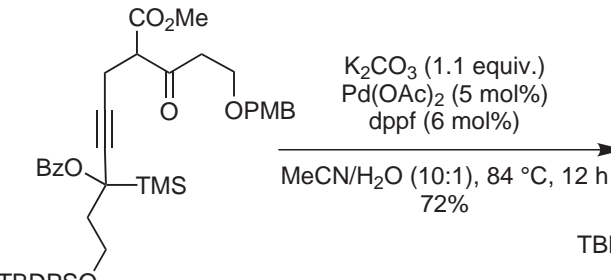

2 Examples are reported including the synthesis of the $\mathrm{C} 1-\mathrm{C} 18$ segment of lophotoxin and pukalide.

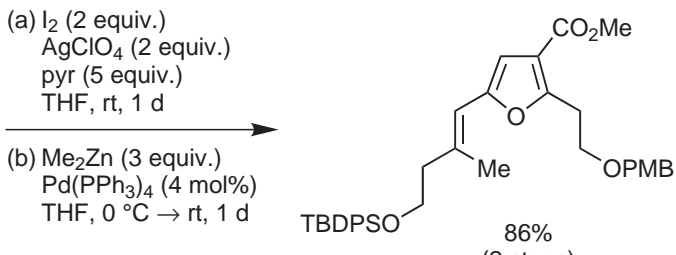

(2 steps)

Tandem carbonyl ylide formation-cycloaddition

Hodgson, D. M.; Avery, T. D.; Donohue, A. C. Org. Lett. 2002, 4, 1809.

Dipolar Cycloaddition

$\overbrace{\mathrm{Me}}^{\mathrm{U}_{\mathrm{Me}}^{\mathrm{N}}}$

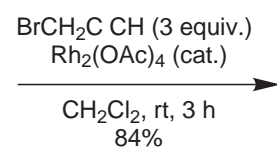

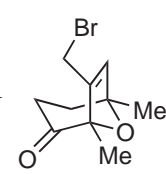
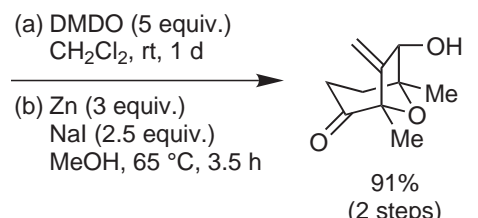

The stereoselective synthesis of cis-nemorensic acid is also reported.

Stereocontrolled synthesis of (+)-biotin from L-cysteine.

Seki, M.; Hatsuda, M.; Mori, Y.; Yamada, S. Tetrahedron Lett., 2002, 43, 3269.

Cyanosilylation

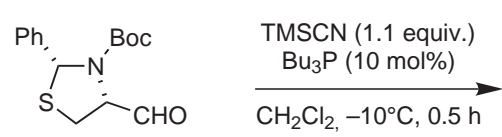<smiles>COC(C)C1CSC(c2ccccc2)N1C(C)C</smiles>

$96 \%$ syn:anti $=92: 8$

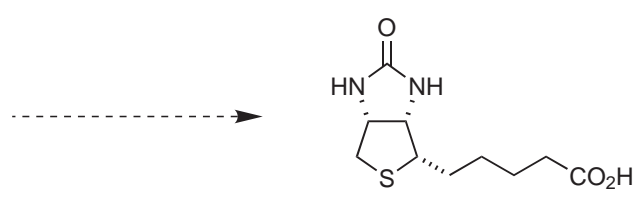

(+)-biotin

1 Example. The product is an intermediate in the synthesis of the natural product (+)-biotin. 